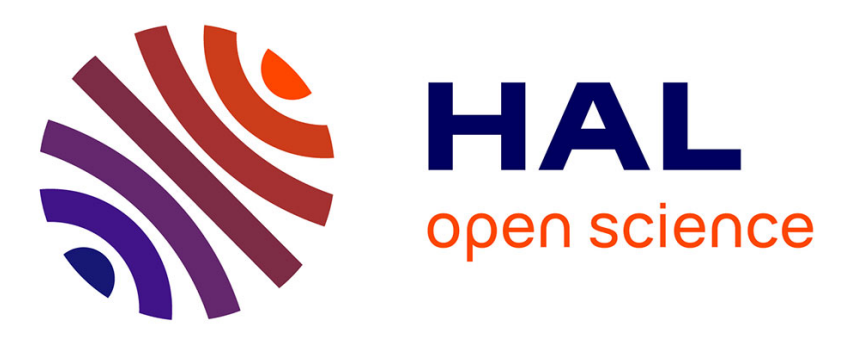

\title{
A controlled quantum SWAP logic gate in a 4-center metal complex
}

Mohamed Hliwa, Jacques Bonvoisin, Christian Joachim

\section{To cite this version:}

Mohamed Hliwa, Jacques Bonvoisin, Christian Joachim. A controlled quantum SWAP logic gate in a 4-center metal complex. Nicolas Lorente, Christian Joachim. Architecture and Design of Molecule Logic Gates and Atom Circuits, Springer, pp.237-247, 2012, Advances in Atom and Single Molecule Machines, 978-3-642-33137-4. 10.1007/978-3-642-33137-4_18. hal-01737292

\section{HAL Id: hal-01737292 \\ https://hal.science/hal-01737292}

Submitted on 19 Mar 2018

HAL is a multi-disciplinary open access archive for the deposit and dissemination of scientific research documents, whether they are published or not. The documents may come from teaching and research institutions in France or abroad, or from public or private research centers.
L'archive ouverte pluridisciplinaire $\mathbf{H A L}$, est destinée au dépôt et à la diffusion de documents scientifiques de niveau recherche, publiés ou non, émanant des établissements d'enseignement et de recherche français ou étrangers, des laboratoires publics ou privés. 


\title{
A controlled quantum SWAP logic gate in a 4-center metal complex
}

Mohamed Hliwa, Jacques Bonvoisin and Christian Joachim

\begin{abstract}
A monomolecular four center low spin paramagnetic organometallic complex is proposed and theoretically studied to work as a controlled quantum swap molecule logic gate. The magnetic super-exchange interaction between the 2 intramolecular qubits depends on the oxydation state of a third intermediate center itself controlled by an intervalence electron transfer process. A model system is build up using entangled spin qubits in the framework of an Heisenberg-Dirac-Van Vleck like spin Hamiltonian demonstrating the effective swapping operation of this complex.
\end{abstract}

\section{Introduction}

Interconnected in an electrical circuit, single molecules can enter in the construction of a calculating unit [1]. A single molecule can also be the calculating unit by itself either in a semi-classical $[2,3]$ or in a quantum like architecture approach [4]. For a quantum like architecture, the input data can be encoded either in the initial quantum state [5] or in the Hamiltonian [6] of the electronic states of the molecule. For the former, the qubits are positioned along the molecular board.

Mohamed Hliwa

Faculty of Sciences Ben M'Sik, University Hassan II-Mohammedia-Casablanca, BP 7955-Sidi Othman, Casablanca, Maroc. Nanoscience Groupe and MANA Satellite, CEMES/CNRS, 29 rue Jeanne Marvig, 31055, Toulouse, France. e-mail: hliwa@cemes.fr

Jacques Bonvoisin

Nanoscience Groupe and MANA Satellite, CEMES/CNRS, 29 rue Jeanne Marvig, 31055,

Toulouse, France. e-mail: bonvoisin@cemes.fr

Christian Joachim

Nanoscience Groupe and MANA Satellite, CEMES/CNRS, 29 rue Jeanne Marvig, 31055,

Toulouse, France. e-mail: joachim@ cemes.fr 
The quantum inputs are encoded in specific non stationnary states of the molecule electronic structure. For performing a quantum computation, Rabi like oscillations result which will fade out due to decoherence and relaxation processes [7]. In the time interval between the initial qubit preparation and the progressive involvement of a decoherence process, it is therefore important to control when to start and when to stop the quantum computation.

Let us consider the simple example of an interacting 2 qubits system described by the hamiltonian $H=H_{0}+\lambda I$ defined on the $\{|i j\rangle\}_{i, j=0,1}$ canonical basis set where $I$ describes the qubit interactions. When $\lambda$ is no zero, a swapping operation $U_{S}$ on the 2 qubits is defined by $U_{S}|i j\rangle=|j i\rangle[8]$. Over the $t_{n}$ time series, $U_{S}=\exp \left(-i \frac{H}{\hbar} t\right)$ for $t=t_{n}$. When $\lambda$ is set to zero the $\{|i j\rangle\}$ basis set is the $H$ eigenbasis set and there is no swapping. Therefore to control a swapping operation, the solution presented in this letter is to switch ON and OFF the interactions between the qubits using an external stimulus. Mixed valence organo-metallic complex molecules are very well adapted for such a control swapping. First, an organometallic complexe can be prepared in low spin oxydation states. Under a static magnetic field, this defines a set of qubits each localized on one metallic center [9]. Second, through bond magnetic superexchange interactions along the organic board of the organo-metallic molecule mediate the quantum information between the qubits with a moderate interaction decay between them as a function of the board length [10]. Third, intramolecular electron transfer processes are known to control the magnetic super exchange interaction [14], a nice way to control the swapping.

In this letter, a generic 4-metal center organometallic mixed valence complex is proposed able to perform a swapping operation controlled by light. After the initial preparation of the intramolecular qubits system, the swap can be initiated at will and stop before the decoherence by a reverse intramolecular electron transfer process. In the subsection 2.1, the chemical structure, the swapping process and its control on this 4-center organometallic complex are presented. In the subsection 2.2 , the Hilbert space to model this 3-center/3-electron system is presented and in subsection 2.3 the corresponding valence bond like magnetic Hamiltonian. In section 3, this Hamiltonian is used to demonstrate how the swapping is performed when the qubit magnetic interactions is $\mathrm{ON}$.

\section{The swaping molecule gate structure and design}

\subsection{The controlled swapping mechanism}

The structure of a controlled swap molecule is presented in figure 1. In this 4 metal center organometallic complex, the $\mathbf{M}_{\mathbf{1}}$ and $\mathbf{M}_{\mathbf{2}}$ sites are carrying the two qubits which are paramagnetic Low Spin (LS) within a doublet ground state. $M_{1}^{n+}$ and $M_{2}^{n+}$ are stabilized in their respective oxidation state by their own coordination sphere. 
They are in magnetic interaction via conjugated organic bridges, through a third central metal ion $M_{3}^{m+}$. This through bond super-exchange magnetic interaction can be switched $\mathrm{ON}$ and $\mathrm{OFF}$ depending on the $\mathbf{M}_{\mathbf{3}}$ oxidation state. This state can be changed from $m=n$ (doublet electronic ground state) to $m=n-1$ (singlet electronic ground state) by exciting the Inter-Valence Transition (IVT) (or Metal-Metal Charge Transfer (MMCT)) band of the lateral $\mathbf{M}_{\mathbf{3}}-\mathbf{M}_{\mathbf{4}}$ mixed valence part of this 4 center complex. $\mathbf{M}_{\mathbf{3}}$ is the control swapping site of the gate.
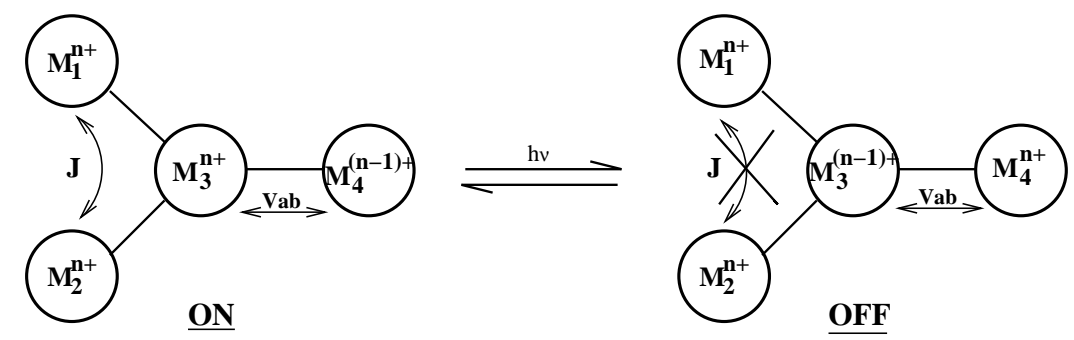

Fig. 1 The model structure of a 4-center controlled swap organometallic complex. The magnetic interaction between the metal centers $\mathbf{M}_{\mathbf{1}}$ and $\mathbf{M}_{\mathbf{2}}$ can be switched ON or OFF depending on the oxidation state of $\mathbf{M}_{\mathbf{3}}$ which changes respectively from $n$ to $(n-1)$ under electron transfer between $\mathbf{M}_{\mathbf{3}}$ and $\mathbf{M}_{\mathbf{4}}$ induced by a specific light radiation. "OFF" is the initial ground state configuration and "ON" the swapping state of the molecule. $V_{a b}$ is the electronic through bond interaction between $\mathbf{M}_{\mathbf{3}}$ and $\mathbf{M}_{\mathbf{4}}$ in their respective oxidation states. $J$ is the spin super-exchange interactions between $\mathbf{M}_{1}$ and $\mathbf{M}_{\mathbf{2}}$ centers through $\mathbf{M}_{\mathbf{3}}$.

When constituted of four identical metallic centers $\mathbf{M}$, the special feature of this molecular structure is that the coordination spheres around each metal ion can be different by chosen different substituents on the surrounding ligands opening a possible optical selective excitation of each center to prepare a given qubits state superposition. A realistic chemical structure of this swap molecule is presented in figure 2 where $\mathbf{M}$ is a ruthenium. As required, this molecule is a non symmetric tetranuclear complex where all the peripheral ruthenium entities are different from each other due to their different coordination spheres $\left(R_{1} \neq R_{2} \neq R_{4}\right)$. Several necessary chemical steps towards this target molecules have been made $[11,12,13]$

\subsection{The model electronic basis set}

Each external metal ion $M_{1}^{n+}$ and $M_{2}^{n+}$ of the complex hold an unpaired electron ( $s=$ $\left.\frac{1}{2}, m_{s}= \pm \frac{1}{2}\right)$ spin state. Under a static external magnetic field, each defines a well localised qubit which can be prepared in a quantum superposition $\alpha\left|\frac{1}{2}\right\rangle+\beta\left|-\frac{1}{2}\right\rangle$. When $M_{3}$ is in its $(n-1)+$ oxidation state, the magnetic interaction between $M_{1}^{n+}$ and $M_{2}^{n+}$ is negligible and the two qubits are not in interaction and each qubit center can be prepared separatly. When the $M_{3}$ is switched on in its $n+$ oxidation state, 


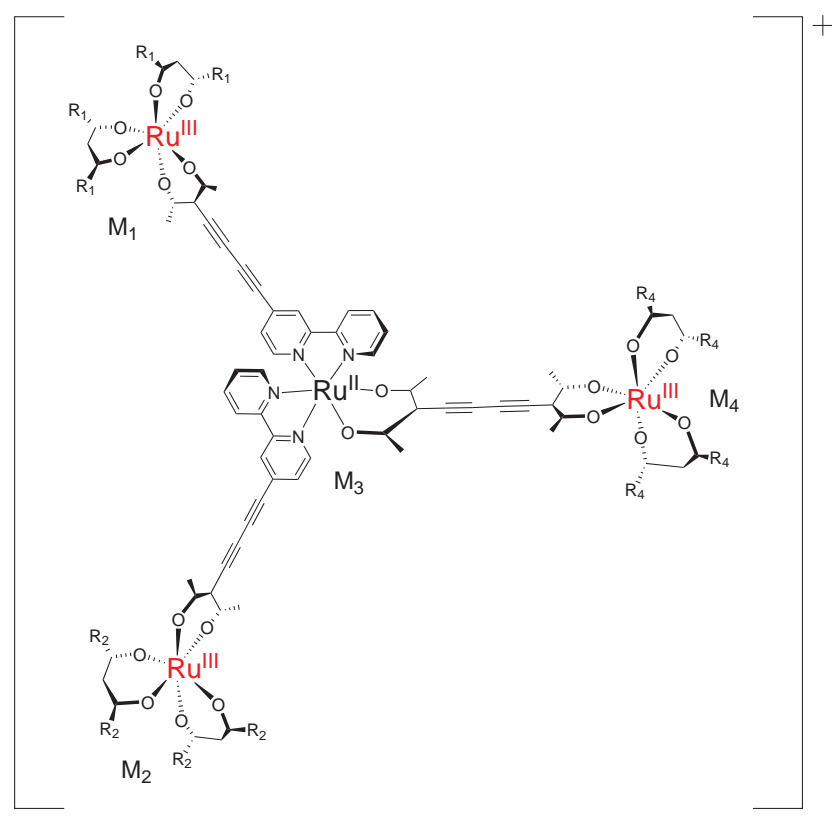

Fig. 2 A possible chemical structure of the proposed swap molecule whose controlled swapping process is presented in this letter.

$M_{1}^{n+}$ and $M_{2}^{n+}$ are magnetically coupled through the organic ligands via $M_{3}^{n+}$ and the swapping begin [14].

Thus, to model this swapping operation, we have to consider the spin interaction between two unpaired electrons each one localized on one metal of two bonded centers, namely $M_{1}^{n+}-M_{3}^{n+}$ and $M_{2}^{n+}-M_{3}^{n+}$. The spin interaction between the electrons on sites $\mathbf{M}_{\mathbf{1}}$ and $\mathbf{M}_{\mathbf{2}}$ is described by an effective magnetic coupling by considering only two 1-qubits, one on each of the two target centers. The initial state is prepared within two paired electrons on the site $\mathbf{M}_{\mathbf{4}}$ and a single electron on each one of the sites $\mathbf{M}_{\mathbf{1}}, \mathbf{M}_{\mathbf{2}}$ and $\mathbf{M}_{\mathbf{3}}$. Therefore, to construct the basis set describing the swapping process, only 3 active centers have to be considered that is the 3 paramagnetic sites $\mathbf{M}_{1}, \mathbf{M}_{2}$ (the qubits sites) and $\mathbf{M}_{3}$ (the control site).

Electron indicernability imposes the use of a Slater determinant canonical basis set:

$$
\{|101\rangle,|011\rangle,|100\rangle,|010\rangle,|001\rangle,|110\rangle\}
$$

to model the swaping operation on this 3-electron/3-active sites electronic system.

The swap mechanism using the $\mathbf{M}_{\mathbf{1}}-\mathbf{M}_{\mathbf{2}}$ spin super-exchange mechanism is represented in figure 3 for each possible canonical preparation state. The intermediate states described by the determinants $|110\rangle$ and $|001\rangle$ correspond to states in which the spin of the qubit sites have the same $m_{s}$ value. The preparation state can be one of the states represented by one of the determinants at the left or at the right in fig- 
ure 3 and the final state is its antagonist. To model the swapping, the model Hilbert space generated by those 6 determinants can be partitioned into two subspaces :

(i) A model subspace which contains the configurations where the qubit sites $\mathbf{M}_{\mathbf{1}}$ and $\mathbf{M}_{\mathbf{2}}$ have spins with the opposite signs and for which the control site $\mathbf{M}_{\mathbf{3}}$ have either $\alpha$ or $\beta$ spin.

$$
\{|010\rangle,|100\rangle,|011\rangle,|101\rangle\}
$$

(ii) an intermediate subspace which contains the configurations for which the qubit sites have spins oriented in the same direction and where the control site have either $\alpha$ or $\beta$ spin.

$$
\{|110\rangle,|001\rangle\}
$$

Whatever the electron spin state on the $\mathbf{M}_{\mathbf{3}}$ site and to prepare an initial doublet state $\left(S=\frac{1}{2}\right)$ of type $|\mathbf{0 1 0}\rangle$ or $|\mathbf{0 1 1}\rangle$, there is a probability $\left|C_{0}\right|^{2}$ to form $|\mathbf{0 1 0}\rangle ;\left(m_{s}=\right.$ $\left.-\frac{1}{2}\right)$ and a probability $\left|C_{1}\right|^{2}$ to form $|\mathbf{0 1 1}\rangle ;\left(m_{s}=\frac{1}{2}\right)$. Because of the degeneracy of those 2 states and by symmetry $\left|C_{0}\right|^{2}$ and $\left|C_{1}\right|^{2}$ can be taken equal to $\frac{1}{2}$.

Those six configurations (micro-states) are two by two maximally quantum entangled so that instead of (2) and (3), two other subspaces of our model Hilbert space can be defined : a swaping subspace generated by (4) and the control sub-space generated by (5):

$$
\begin{gathered}
\left\{\begin{array}{c}
\frac{1}{\sqrt{2}}[|101\rangle \pm|100\rangle]=\left|\Phi_{M_{1} \bar{M}_{2}}^{g, u}\right\rangle \\
\frac{1}{\sqrt{2}}[|011\rangle \pm|010\rangle]=\left|\Phi_{\bar{M}_{1} M_{2}}^{g, u}\right\rangle
\end{array}\right. \\
\frac{1}{\sqrt{2}}[|110\rangle \pm|001\rangle]=\left|\Phi_{M_{1} M_{2}, \bar{M}_{1} \bar{M}_{2}}^{g, u}\right\rangle
\end{gathered}
$$

They are expressed as symmetric and antisymmetric linear combinations of the entangled components of the above defined model and intermediate spaces. Note that those new symmetric and anti-symmetric states are orthonormal and consequently not coupled together by symmetry. This allows to describe the qubits superexchange interactions by a 2-bloc diagonal hamiltonian built separately on the symmetric $g$ and on the anti-symmetric $u$ parts.

\subsection{The controlled swap hamiltonian in the interaction picture}

In absence of an external magnetic field and when the spin-orbit interactions are neglected, the interaction between two or more paramagnetic centers can be described through spin operators which acts only on local effective spin eigenfunctions and therefore represented on the basis set of its localized spin eigenstates. In a such case, the so-called Heisenberg-Dirac-Van Vleck spin-hamiltonian $H_{H D V V}$ $[15,16,17]$ is the most commonly used. Many formulations of the $H_{H D V V}$ can be 


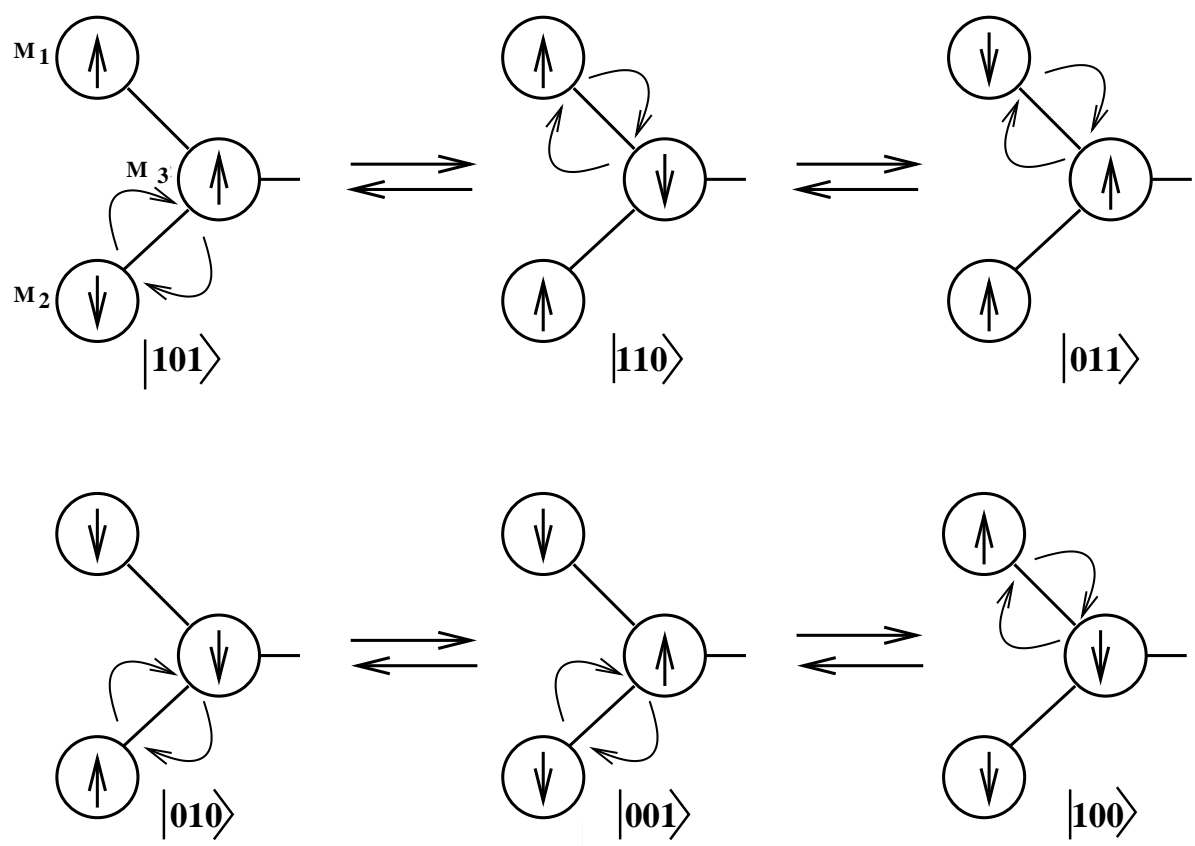

Fig. 3 schematic representation of the swapping mechanism

founded in literature $[18,19,20]$, it is a pair interaction operator and usually it takes the form :

$$
\hat{H}_{H D V V}=\sum_{i>j}-J\left(\overrightarrow{r_{i j}}\right) \hat{\overrightarrow{S_{i}}} \cdot \hat{\overrightarrow{S_{j}}}
$$

where $J$ is the spin exchange coupling constant, generally depending on the distance $r_{i j}$ between two electrons localized on centers $i$ and $j$. Let us consider the two qubit magnetic sites $\mathbf{M}_{\mathbf{1}}$ and $\mathbf{M}_{\mathbf{2}}$ so distant from each other that they do not interact through space directly. Each one is through ligand interacting with the third magnetic site $\mathbf{M}_{\mathbf{3}}$ (control site). In such case, we can write

$$
\left\{\begin{array}{c}
J=J_{13}=J_{23} \\
J_{12}=0
\end{array}\right.
$$

between the spin exchange constants and $\hat{H}_{H D V V}$ can be simply rewriten:

$$
\hat{H}_{H D V V}=-J\left(\hat{S}_{1} \hat{S}_{3}+\hat{S}_{2} \hat{S}_{3}\right)
$$

Then, if one uses the 6 Slater determinants in (2) and (3) plus the two determinants $|000\rangle$ and $|111\rangle$ corresponding to the degenerated and non-interacting quadruplet states, a $(8 \times 8) H D V V$ hamiltonian matrix can be constructed to describe our magnetic system. This matrix is bloc-diagonal and contains two diagonal elements 
which correspond to the pure quadriplet states and two $(3 \times 3)$ bloc matrices involving configurations (micro-states) which are doublets formed by two electrons holding the same spin and the third one with a spin of the opposite orientation. Inside the $(3 \times 3)$ bloc built on the partial basis set $\{|001\rangle,|010\rangle,|100\rangle\}$, the configurations $|010\rangle$ and $|100\rangle$ are not directly coupled but are mutually interacting via the configuration $|001\rangle$. In the second $(3 \times 3)$ bloc, the interaction between the configurations $|011\rangle$ and $|101\rangle$ is also passing through the configuration $|110\rangle$. This is resulting from two successive permutations between spin of opposite signs, firstly for a single occupied magnetic sites $\mathbf{M}_{\mathbf{1}}$ and $\mathbf{M}_{\mathbf{3}}$ (or $\mathbf{M}_{\mathbf{2}}$ and $\mathbf{M}_{\mathbf{3}}$ ) and secondly a spin permutation ( or a spin exchange ) between electrons on sites $\mathbf{M}_{\mathbf{3}}$ and $\mathbf{M}_{\mathbf{2}}$ (or $\mathbf{M}_{3}$ and $\mathbf{M}_{1}$ ). The first spin exchange operation gives rise to an intermediate microstate in which the spin momentum at sites $\mathbf{M}_{\mathbf{1}}$ and $\mathbf{M}_{\mathbf{2}}$ have the same $m_{s}$ value and the control site $\mathbf{M}_{\mathbf{3}}$ has an $m_{s}$ value with the opposite sign of its initial value. The second operation restores the $m_{s}$ value on the magnetic site $\mathbf{M}_{\mathbf{3}}$ and performs the complete interchange between the sign-opposite $m_{s}$ values on the target sites $\mathbf{M}_{\mathbf{1}}$ and $\mathbf{M}_{2}$. the relative energy of the intermediate micro-states is equal to $+\frac{\mathbf{J}}{\mathbf{2}}$ and the magnetic coupling exchange energy is equal to $-\frac{\mathrm{J}}{2}$. Diagonalization of this $H D V V$ matrix will provide 8 eigenstates corresponding to 8 non-entangled quantum states which are 2 by 2 degenerated and consequently the configurational mixed states define four doubly degenerated energy levels.

On this magnetic Hamiltonian, the effect of an external static magnetic field $\overrightarrow{B_{z}}$ can be modeled by the hamiltonian: (9)

$$
\begin{aligned}
& \hat{H}_{B}=\mu_{B} \hat{\vec{B}}_{z} \cdot \hat{\vec{S}}=\mu_{B} \hat{B}_{z} \hat{S}_{z} \\
& \quad=\Lambda_{z} \hat{S}_{z}=\Lambda_{z}\left(\hat{S}_{1_{z}}+\hat{S}_{2_{z}}+\hat{S}_{3_{z}}\right)
\end{aligned}
$$

where $\mu_{B}, \hat{B}_{z}$ and $\hat{S}_{i_{z}}$ are respectively the Bohr magneton, the quantification $z-$ axis projection of the magnetic field vector and of the spin momentum localized at a given magnetic site $\mathbf{M}_{\mathbf{i}}$. By introducing (9) in (8), one obtains a new $H_{H D V V}$ Hamiltonian matrix in which the doubly degeneracy in each $(3 \times 3)$-bloc diagonal sub-matrix are removed as it is shown in the matrix given in (10). The effect of $\overrightarrow{B_{z}}$ is to shift the energies of the matrix diagonal elements and consequently to remove the degeneracies between each pair of the $H_{H D V V}$ eigenstates. The final $H_{H D V V}+H_{B}$ $(8 \times 8)$-Hamiltonian matrix is given by: 


\begin{tabular}{|c|c|c|c|c|c|c|c|c|}
\hline & $|000\rangle$ & $|001\rangle$ & $|010\rangle$ & $|100\rangle$ & $|011\rangle$ & $|\mathbf{1 0 1}\rangle$ & $|\mathbf{1 1 0}\rangle$ & $|111\rangle$ \\
\hline$|000\rangle$ & $-\frac{J-3 \Lambda_{z}}{2}$ & 0 & 0 & 0 & 0 & 0 & 0 & 0 \\
\hline$|001\rangle$ & 0 & $+\frac{J+\Lambda_{z}}{2}$ & $-\frac{J}{2}$ & $-\frac{J}{2}$ & 0 & 0 & 0 & 0 \\
\hline$|010\rangle \mid$ & 0 & $-\frac{J}{2}$ & $\frac{\Lambda_{z}^{2}}{2}$ & 0 & 0 & 0 & 0 & 0 \\
\hline$|\mathbf{1 0 0}\rangle$ & 0 & $-\frac{J}{2}$ & 0 & $\frac{\Lambda_{z}}{2}$ & 0 & 0 & 0 & 0 \\
\hline$|011\rangle$ & 0 & 0 & 0 & 0 & $-\frac{\Lambda_{z}}{2}$ & 0 & $-\frac{J}{2}$ & 0 \\
\hline$|101\rangle$ & 0 & 0 & 0 & 0 & 0 & $-\frac{\Lambda_{z}}{2}$ & $-\frac{J}{2}$ & 0 \\
\hline$|\mathbf{1 1 0}\rangle\rangle$ & 0 & 0 & 0 & 0 & $-\frac{J}{2}$ & $-\frac{J}{2}$ & $+\frac{J-\Lambda_{z}}{2}$ & 0 \\
\hline$|\mathbf{1 1 1}\rangle \mid$ & 0 & 0 & 0 & 0 & 0 & 0 & 0 & $-\frac{J+3 \Lambda_{z}}{2}$ \\
\hline
\end{tabular}

In the framework of the Heisenberg interaction representation, this is the valence bond like Hamiltonian matrix used in the next section to get the swapping Hamiltonian matrix on the canonical basis set of the 3-qubit supporting the swap logic function between the two qubits at the magnetic sites $\mathbf{M}_{\mathbf{1}}$ and $\mathbf{M}_{\mathbf{2}}$ controlled by the qubit at the site $\mathbf{M}_{\mathbf{3}}$. The spin super-exchange mechanism between the site $\mathbf{M}_{\mathbf{1}}$ and $\mathbf{M}_{\mathbf{2}}$ is performed in two steps. A super-exchange occurs firstly between one of the target sites $\mathbf{M}_{\mathbf{1}}$ (or $\mathbf{M}_{\mathbf{2}}$ ) and the control site $\mathbf{M}_{\mathbf{3}}$. The second step consists in a spin permutation between the site $\mathbf{M}_{3}$ and the other qubit site $\mathbf{M}_{\mathbf{2}}$ (or $\mathbf{M}_{\mathbf{1}}$ ). For its initial and final states, a two step transformation has a configuration where the two spins localised on each qubit site have an opposite sign $m_{s}$ value $\left(+\frac{1}{2}\right.$ or $\left.-\frac{1}{2}\right)$, but has an intermediate state configuration within spins on the target sites having the same $m_{s}$ value.

\section{The time dependent swapping operation}

Since the swap system is only defined on the doublet states, one need, for example, to use the symmetric determinant combinations $g$. In this case, the final model Hilbert space is generated by the three determinants $\left|\Phi_{M_{1} \bar{M}_{2}}^{g}\right\rangle,\left|\Phi_{\bar{M}_{1} M_{2}}^{g}\right\rangle$ and $\left|\Phi_{M_{1} M_{2}, \bar{M}_{1} \bar{M}_{2}}^{g}\right\rangle$ given in (4) and (5). Describing the swaping process and given in $J$ unit, the corresponding $(3 \times 3)$-block Hamiltonian matrix coming from (11) can be written on this basis set:

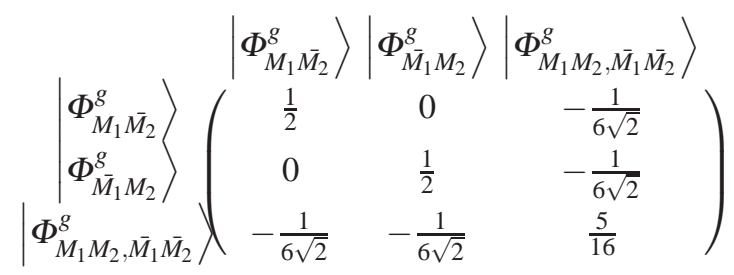


Notice that (11) is free from the static magnetic field contribution. The total $\hat{S}_{z}$ operator action on each state of the final swap space gives zero as eigenvalue. On each site, the role played by the magnetic field is only to lift up the degeneracy between the $\alpha$ and $\beta$ electron spins so that the swapping process can be initiated on well determined qubits inside the molecule with no direct influence on the process itself.

To determine the swap oscillation frequency and the time-dependent probability to find the system in a given quantum state, we have solved the time-dependent Schrödinger equation using (11). In this representation, the Rabi oscillation frequency between, for instance, an initial state $\left|\phi_{i}\right\rangle=\left|\Phi_{M_{1} \bar{M}_{2}}^{g}\right\rangle$ and a final state $\left|\phi_{f}\right\rangle=\left|\Phi_{\bar{M}_{1} M_{2}}^{g}\right\rangle$ is given by their effective magnetic coupling via the intermediate state $\left|\phi_{m}\right\rangle=\left|\Phi_{M_{1} M_{2}, \bar{M}_{1} \bar{M}_{2}}^{g}\right\rangle$. The calculation of this effective coupling is performed by partitionning the total active space Hamiltonian $H$ given in (11) as $H=P H P+Q H Q+P H Q+Q H P$, where $P=\left|\phi_{i}\right\rangle\left\langle\phi_{i}|+| \phi_{f}\right\rangle\left\langle\phi_{f}\right|$ and $Q=\left|\phi_{m}\right\rangle\left\langle\phi_{m}\right|$ are the projectors respectively defined on the qubits and the control subspaces. Then, starting from a given initial state, the time-dependent probability to find the system in a final state is given by $\mathscr{P}_{f}(t)=\left|\left\langle\phi_{f} \mid \psi(t)\right\rangle\right|^{2}$, where $|\psi(t)\rangle=\sum_{n} C_{n}(t)\left|\psi_{n}\right\rangle$ and $\left|\psi_{n}\right\rangle$ are the (11) eigenvectors. The time-dependent quantum evolution of this 3state swap system results from the superposition of 3 sinusoidals whose frequencies can be calculated by the 2-by-2 difference of the eigenvalues and their respective weight by using the time dependent population Fourier transform. The fundamental frequency of the corresponding Fourier spectrum can also be extracted using the effective Hamiltonian $H^{e f f}=P U^{-1} H U P$ expressed on the basis set associated to the qubits $\mathrm{P}$-space where $U$ is a non unitary transformation which bloc-diagonalize the total swap Hamiltonian $H$ [21]. Using this transformation, the calculated effective magnetic coupling $H_{i f}^{e f f}=\frac{\left|E_{f}^{e f f}-E_{i}^{e f f}\right|}{2}$ between the 2 qubits through the central control site is equal to 0.0497 (in $J$ unit). The corresponding calculated time-dependent $(i \rightarrow f)$ oscillation probabilities are reported in figure 4 . The first maximum of $\mathscr{P}_{f}(t)$ giving the fundamental swap oscillation frequency is reached at the time $t=\frac{\pi \hbar}{2 H_{i f}^{e f f}}$ that is $31.562 \frac{\hbar}{J} \mathrm{sec}$. As an example, for a standard $J=100 \mathrm{~cm}^{-1}$ [22], the swapping oscillation frequency is $597 \mathrm{GHz}$.

As compared to a non-controlled swapping system where the 2 qubits are directly through space interacting to operate a swap, our intermediate control site is not perturbing a lot the swapping operation. The time dependent evolution is a little deformed by the presence of the controlling intermediate site. But the 2 additional frequency components in the swapping time dependent quantum process have a small enough contribution to preserve a nice Rabi like oscillation of the swapping in time as presented in figure 4.

In conclusion, we have proposed a molecule and a model to implement a quantum controlled swap molecule logic gate. The swapping process involves 3 paramagnetic adjacents centers, the preparation of the active sites is firstly initiated by switching 
ON an appropriate light radiation to transfert an electron from the controlling site to the passive $4^{\text {th }}$ center. The active qubits formation is initiated by applying an static magnetic field. This external static magnetic field is necessary to define the qubits on the molecule but its magnitude do not influences the swapping time. From an experimental point of view, several techniques are under development to detect [5, 23], adress [24], flip [25], visualize [26] or probe [27] single spins on a molecule with an enough spatial resolution to input the quantum information on our swap molecule logic gate. It remains to adapt the design of the presented molecule for the swapping operation to work on a surface.
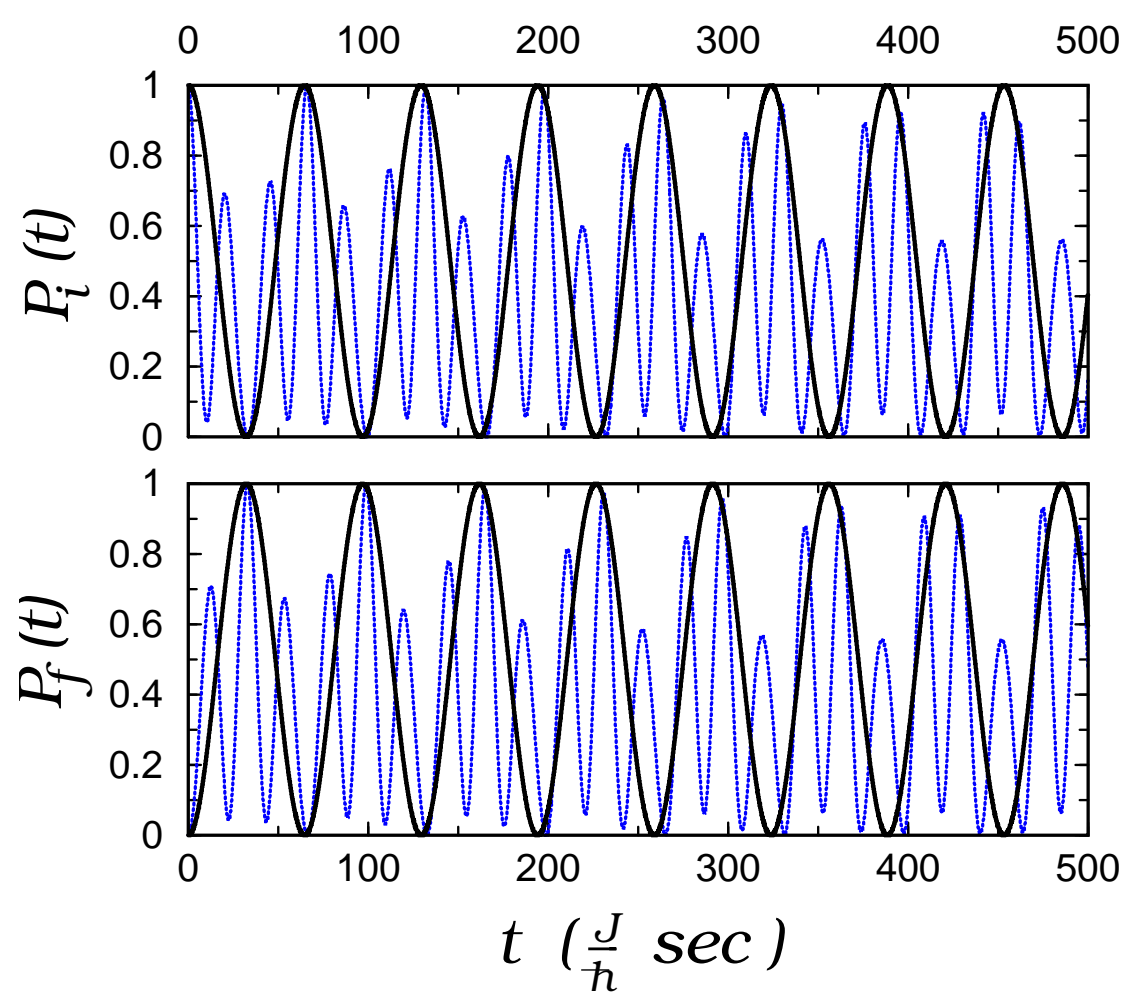

Fig. 4 The controlled swap time-dependent probability. $\mathscr{P}_{f}(t)$ and $\mathscr{P}_{i}(t)$. These Rabi oscillations curves are obtained by using $H$ (solid lines) or $H_{\text {eff }}$ (dashed lines).

\section{Acknowledgement}

The authors like to thank MANA for financial support via the WPI Program. 


\section{References}

[1] Stadler, R., Ami, S., M. Forshaw, M., Joachim, C. : Integrating logic functions inside a single molecule. Nanotechnology 15, S115 (2004).

[2] Carter, F. L. : Molecular Electronic Devices II. ed. Dekker, New York (1987) : The molecular device computer : Point of departure for large scale cellular automata. Physica D10, 175 (1984).

[3] Jlidat, N., Hliwa, M., Joachim, C. : A semi-classical XOR logic gate in a single molecule. Chem. Phys. Lett. 451, 270 (2008).

[4] Duchemin, I., Renaud, N., Joachim, C. : An intermolecular 1/2-adder with tunneling current drive and read-outs. Chem. Phys. Lett. 452, 269 (2008).

[5] Feng, M., Twamley, J. : Single spin detection by qubit swap to molecular nanomagnet. Europhys. Lett. 69, 699 (2005).

[6] Duchemin, I., Joachim, C. : A quantum digital half adder inside a single molecule. Chem. Phys. Lett. 406, 167 (2005).

[7] Bertaina, S., Gambarelli, S., Mitra, T, Tsukerblat, B., Müller, A., Barbara, B. : Quantum oscillations in a molecular magnet. Nature 453, 203 (208).

[8] Liu, J., Zhang, G.-F., Chen, Z.-Y. : Influence of magnetic field on swap operation in Heisenberg XXZ model. Physica B 404, 1116 (2009).

[9] Fabre, M., Jaud, J., Hliwa, M., Launay, J.-P., Bonvoisin, J. : On the role of the bridging dicyanamidobenzene ligand in a new binuclear ruthenium complexe. Inorg. Chem. 45, 9332 (2006).

[10] Fabre, M., Bonvoisin, J. : Electronic and magnetic communication in mixed-valent and homovalent ruthenium complexes containing phenylcyanamide type bridging ligands. J. Am. Chem. Soc. 129, 1434 (2007).

[11] Munery, S., Ratel-Ramond, N., Benjalal, Y., Vernisse, L., Guillermet, O., Bouju, X., Coratger, R., Bonvoisin, J. : Synthesis and characterization of a series of ruthenium tris( $\beta$-diketonato) complexes by an UHV-STM investigation and numerical calculations. Eur. J. Inorg. Chem. 2698 (2011).

[12] Viala, C., Bonvoisin, J. : Synthesis and characterization of $\beta$-diketonato ruthenium(II) complexes with two 4-bromo or protected 4-ethynyl-2,2'-bipyridine ligands. Inorg. chim. Acta 363, 1409 (2010).

[13] Munery, S., Jaud, J., Bonvoisin, J. : Synthesis and characterization of bis(bipyridine) ruthenium(II) complexes with bromo and protected ethynyl $\beta$-diketonato ligands. Inorg. Chem. Commun. 11, 975 (2008).

[14] Journeaux, Y., Sletten, J., Kahn, O. : Tunable interaction in ( $\mu$-oxamido) copper(II) binuclear complexes. Inorg. Chem. 25, 439 (1986).

[15] Heisenberg, W. : Zur theorie des ferromagnetismus. Z. Phys. 49, 619 (1928).

[16] Dirac, P. A. M. : Quantum mechanics of many-electron systems. Proc. R. Soc. London A123, 714 (1929)

[17] Van Vleck, J. H. : The theory of electric and magnetic susceptibility. Oxford University Press, Oxford (1932).

[18] Calzado, C. J., Cabrero, J., Malrieu, J.-P., Caballol, R. : Analysis of magnetic coupling in binuclear complexes. I and II- derivation of valence effective Hamiltonian from ab-inition CI and DFT calculations. J. Chem. Phys. 116, 2728 (2002), J. Chem. Phys. 116, 3985 (2002).

[19] Doublet, M.-L., Lepetit, M.-B. : Correlation and dimerization effects on the physical behaviour of $\mathrm{NR}_{4}\left[\mathrm{Ni}(\mathrm{dmit})_{2}\right]_{2}$ charge transfer salts : density matrix renormalization group study of the quarter-filling t-J model. J. Chem. Phys. 110, 1767 (1999).

[20] Capelle, K., Libero, V.-L. : Spin density functional theory : Some open problems and application to inhomogeneous Heisenberg model. Int. J. Quant. Chem. 105, 679 (2005).

[21] Durand, Malrieu, J.-P. : Effective Hamiltonian and pseudo-operators as tools for rigorous modelling. Adv. Chem. Phys. 67, 321 (1987).

[22] M. A. S. Aquino, M. A. S., Lee, F., Gabe, L. E. J., Bensimon, C., Greedan, J. E., Crutchley, R. J. : Superexchange metal-metal coupling in dinuclear pentaamine rethenium complexes 
incorporating a 1,4-dicyanamidobenzene dianion bridging ligand. J. Am. Chem. Soc. 114, 5130 (1992).

[23] Rugar, D., Budakian,R., Mamin, R. J. : Single spin detection by magnetic resonance force microscopy. Nature 430, 329 (2004).

[24] Messina, P., Mannini, M., Caneschi, A., Gatteschi, D., Sorace, L., Sigalotti, P., Sandrin, C., Prato, S., Pittana, P., Manassen, Y. : Spin noise fluctuation from paramagnetic molecular adsorbate on surfaces. J. Appl. Phys. 101, 053916 (2007).

[25] Heinrich, A. J., Gupta, J. A., Lutz, C. P., Eigler, D. M. : Single atom-flip spectroscopy. Science 306, 466 (2004).

[26] Iacovita,C.,Rastei, M. V., Heinrich, B. W., Brumme, T., Kortus, J., Limot, L., Bucher, J.P. : Visualizing the spin of individual cobalt-phthalocyanine molecule. Phys. Rev. Lett. 101, 116622 (2008).

[27] Meier, F., Zhou, L., Wiebe, J., Wiesendanger, R. : Revealing magnetic interactions from single-atom magnetization curves. Science 320, 82 (2008). 